\title{
Experimental testing of the sorption-mechanical coupled behaviour of polyethylene into pressurized hydrogen
}

\author{
S. Castagnet ${ }^{\mathrm{a}}$, J.C. Grandidier, M. Comyn and G. Benoît \\ Institut P' - Département de Physique et Mécanique des Matériaux \\ Ensma, 1 Avenue Clément Ader, BP 40109, 86961 Futuroscope cedex, France
}

\begin{abstract}
Coupling between pressurized gas sorption and tensile behavior was studied in a polyethylene (PE). Tensile and creep tests into hydrogen (30 bars) and nitrogen (30 bars) atmosphere were compared to experiments into atmospheric air. Gaseous environment was shown to have noticeable influence neither on the modulus / yield stress in tension, nor on the long term creep behavior. Mechanical consequence of a long term aging into hydrogen atmosphere was also investigated in samples previously exposed to hydrogen at various aging conditions (temperature, pressure, duration). No deleterious effect on the tensile properties of PE was evidenced.
\end{abstract}

\section{INTRODUCTION}

Some applications involving usual polymers imply direct contact with gases expected to transport into the macromolecular network, either for application purpose like in separation gas membranes or gas supplying, or due to the part environment.

Gas transport properties (permeability, diffusivity, solubility) of polymers has been widely investigated in systems made of several gases (among which hydrogen $(\mathrm{H} 2)$ [1,2], oxygen $(\mathrm{O} 2)$ [35], and carbon dioxide (CO2) [4-6]) into several amorphous or semi-crystalline polymers (among which polyethylene (PE) [5-7], polypropylene (PP) [3] or polyimide [2]).

Only a few works considered relationships between gas sorption and mechanical behavior in thermoplastics [7-9]. Even less works have been reported on explicit coupling between diffusion and mechanical behavior into a unique constitutive law, like proposed by Valançon et al. for water diffusion and viscoelasticity [10]. Nevertheless, gas penetration is bound to affect the macromolecular mobility and subsequently the mechanical behavior, with some specificity in semicrystalline systems where gas penetration preferentially occurs in the confined amorphous phase.

For hydrogen, coupling between mechanical behavior and gas diffusion in thermoplastics has not been characterized, probably due to the strong precautions safety care required by hydrogen manipulation. However, the topicality of this issue becomes emphasized by research on the opportunity to make hydrogen a substitutive energy vector. Based on the development of an original mechanical testing device into pressurized hydrogen atmosphere, this work aims at highlighting two aspects of the influence of hydrogen on the tensile behavior of semi-crystalline polymers. It is based on a polyethylene widely used for natural gas transport. On one side, the coupling between

\footnotetext{
a e-mail : sylvie.castagnet@ensma.fr or sylvie.castagnet@ cnrs.pprime.fr
} 
mechanical behavior and hydrogen diffusion is investigated through tensile tests performed under hydrogen pressure. The influence of long-term hydrogen-atmosphere aging on the mechanical behavior is addressed on the other side. The hydrogen pressure was limited to 30 bars consistently with gas supply network standards. As a first evaluation here, the mechanical framework was restricted to tensile and creep properties.

\section{EXPERIMENTAL}

\section{Materials}

A polyethylene 100 (PE) provided by Arkema in the form of $1 \mathrm{~mm}$-thick extruded sheets was tested in the as-received form and after long-term aging into a pressurized hydrogen atmosphere (named "hydrogen-aged" samples in the following). As determined by Differential scanning calorimetry (DSC) (Q1000 TA Instruments standard DSC at $20^{\circ} \mathrm{C} /$ min under $\mathrm{N}_{2}$ sweep) the crystallinity ratio was $57 \%$ and the melting temperature $130^{\circ} \mathrm{C}$. The alpha-c transition corresponding to the onset of mobility of conformational defects into the crystalline lamellae and usually located at $\mathrm{T} \alpha_{\mathrm{c}}=60-80^{\circ} \mathrm{C}$ could not be observed in DSC thermograms.

Tensile tests have been performed in dumbbell specimens machined in the extrusion direction with the geometry summarized in Table 1 . Due to safety reasons, the volume of the hydrogen chamber is small (inner diameter $150 \mathrm{~mm}$; deepness $100 \mathrm{~mm}$ ) and moreover reduced by the grips volume. Samples had to be short enough to keep a sufficient displacement range. "Hydrogen-aged" samples were machined with a hollow punch from the 1-mm thick extruded sheets used in another part of the research program [11] to perform hydrogen permeation experiments. During these experiments, PE samples have been maintained in a $100 \% \mathrm{H}_{2}$ atmosphere during various times (from 1 to 13 months) at three different temperatures $\left(20,50\right.$ and $\left.80^{\circ} \mathrm{C}\right)$ and two differential pressures between the sheet sides ( 5 and 20 bars). Aging conditions are enumerated in Table 1.

\begin{tabular}{|c|c|c|c|c|c|}
\hline as-received & \multicolumn{5}{|c|}{ aged into pressurized hydrogen atmosphere } \\
\hline 35 & \multirow{2}{*}{18} & & Temperature $\left({ }^{\circ} \mathrm{C}\right)$ & Pressure (bars) & Aging time (months) \\
\hline 15 & & V1 & 80 & 20 & 9 \\
\hline \multirow{5}{*}{$\begin{array}{c}80 \\
e=1 \mathrm{~mm}\end{array}$} & \multirow{5}{*}{$\begin{array}{l}50 \\
e=1 \mathrm{~mm}\end{array}$} & $\mathrm{~V} 2$ & 20 & 20 & 13 \\
\hline & & V3 & 80 & 5 & 13 \\
\hline & & V4 & 80 & 20 & 1 \\
\hline & & V5 & 50 & 20 & 13 \\
\hline & & V6 & 20 & 5 & 13 \\
\hline
\end{tabular}

Table 1. Samples geometry and aging history (temperature, pressure and duration).

\section{Mechanical tests under pressure}

An Instron 8802 tensile testing machine has been fitted with a pressurized-hydrogen chamber displaying pressures from the ambient up to 400 bars. The gas chamber could be filled by nitrogen or hydrogen. All tests were carried out at 30 bars at ambient temperature, after equilibration of the sample at the ambient air temperature before the test (about $18^{\circ} \mathrm{C}$ for most tests). The axial force $\mathrm{F}$, axial displacement of the cylinder $\mathrm{d}$, temperature $\mathrm{T}$ and pressure $\mathrm{P}$ were monitored during the test. The axial force was recorded from a $20 \mathrm{kN}$ load cell located between the cylinders. The load cell accuracy is $0.4 \%$ of the full range, i.e. $\pm 10 \mathrm{~N}$.

Tensile tests have been carried out at constant displacement speed of the cylinder, i.e. at constant conventional strain-rate. The Cauchy stress $\sigma$ and the logarithmic strain $\varepsilon$ were calculated from $F, d$, the initial gauge length of the sample and the initial cross section area, assuming a constant volume deformation. The Young's modulus is calculated from a linear interpolation of the stress-strain curves between 0 and $1 \%$ or $2 \%$ for PA11 and PE-based materials respectively. The yield stress was defined as the maximal force point in softening curves and as the onset of the stress-hardening stage in consolidating curves. 
To avoid any dangerous mixture between the filling hydrogen and the oxygen contained in the ambient air after closure of the chamber, three successive pressurization / depressurization purging cycles were performed first, by introducing nitrogen up to 10bars. Then, the chamber is filled with hydrogen at a constant pressure rate of 6 bars/min up to 30 bars. The temperature raise is rather quick at the beginning and progressively equilibrated by the water circulating around the chamber and the load cell.

A constant pressure stage is applied before testing, to equilibrate temperature (about 20 minutes long) and to complete hydrogen sorption into the sample. The time needed to reach hydrogen saturation of the sample was estimated from a two-dimensional calculation through an infinite sheet of constant thickness e. The hydrogen concentration on the top and bottom surfaces of the sheet is deduced from a Henry's law. Solubility is assumed to be temperature independent. Concentration is. The initial hydrogen concentration within the sheet is assumed to be zero. The current global concentration $\mathrm{C}(\mathrm{t})$, expressed as a volume ratio between the sorbed gas and the polymer, in $\mathrm{cm}^{3}$ (STP). $\mathrm{cm}^{-3}$ where STP stands for Standard conditions of Temperature (273K) and Pressure $(0.1013$ $\mathrm{MPa}$ ), is obtained from the analytical solution proposed by Crank [12] based on Fick's theory. Solubility $\left(\mathrm{S}_{\mathrm{g}}=0.036 \mathrm{bars}^{-1}\right)$ and the diffusion coefficient of $\mathrm{H}_{2}$ into $\mathrm{PE}\left(\mathrm{D}=5.6710^{-11} \mathrm{~m}^{2} . \mathrm{s}^{-1}\right)$ were deduced from the permeation investigations performed in the same mono-layered materials in another part of the research program [13]. Time needed for hydrogen saturation into the whole specimen under 30 bars was estimated to one hour for both materials; it appears overestimated since the hydrogen diffusion through the lateral faces of the sample was not taken into account in this twodimensional calculation.

During the loading / unloading stage a constant cylinder displacement was applied, corresponding to a constant conventional strain-rate of $8.610^{-3} \mathrm{~s}^{-1}$ for as-received samples and 1.6 $10^{-2} \mathrm{~s}^{-1}$ for aged samples. The temperature keeps stable all along the mechanical test $\left( \pm 0.1^{\circ} \mathrm{C}\right)$. The chamber was depressurized at a constant pressure rate of $6 \mathrm{bars} / \mathrm{min}$, enhancing a temperature decrease. For safety reasons again, the remaining hydrogen had to be purged by three nitrogen purging cycles at 10 bars before opening the chamber.

Similar tensile tests were carried out in pressurized nitrogen and air at atmospheric pressure. The experimental protocol for nitrogen tests was very close to that above described for hydrogen except that the purging step performed for safety reasons at the end of the test was removed. Thus, the mechanical history was the same as for experiments under hydrogen pressure. Similar sorption calculations were made from diffusion and solubility coefficients for nitrogen into PE [14] showing that the saturation regime was also reached after the one-hour step defined for the hydrogen protocol.

Reproducibility was estimated as a standard deviation from the mean value by repeating the same experiment between 3 and 6 times for each material and loading condition. The reproducibility of experiments in ambient air (including the experimental device precision, the dimensional variability of samples and material heterogeneity) was estimated from tests performed in as-received PE. The axial force scattering measurement over 6 tests was $\pm 3.1 \%$. The experimental error increased for pressure experiments due to sliding effects between cylinders and o-rings: the axial force scattering reached $\pm 10 \%$ (4 tests). A more precise measurement of the mechanical under hydrogen pressure would require larger specimens not really compatible with the small volume of the chamber.

Creep tests were performed by following the same protocol except that the loading / unloading stage was replaced by a 50-min creep stage (constant conventional stress $5 \mathrm{MPa}$ ) followed by a 60min recovery step. 


\section{RESULTS AND DISCUSSION}

\section{Coupling between mechanical properties and hydrogen sorption}

Figure 1 depicts the tensile behavior of as-received PE tested at ambient air, and under a 30 bars pressure of hydrogen and nitrogen. Focusing successively on each gaseous environment, it appears that differences between curve series cannot be dissociated from the experimental scatter. It means that a possible hydrogen effect on the tensile behavior of $\mathrm{PE}$ at $18^{\circ} \mathrm{C}$ does not exceed $10 \%$. The Young's modulus is estimated to $950 \pm 100 \mathrm{MPa}$ (over 6 tests), $970 \pm 70 \mathrm{MPa}$ (over 4 tests) and 980 $\pm 50 \mathrm{MPa}$ (over 5 tests) in atmospheric air, 30 bars hydrogen and 30 bars nitrogen respectively. The slight difference between the first value and the two later ones cannot be regarded as a significant pressure effect.

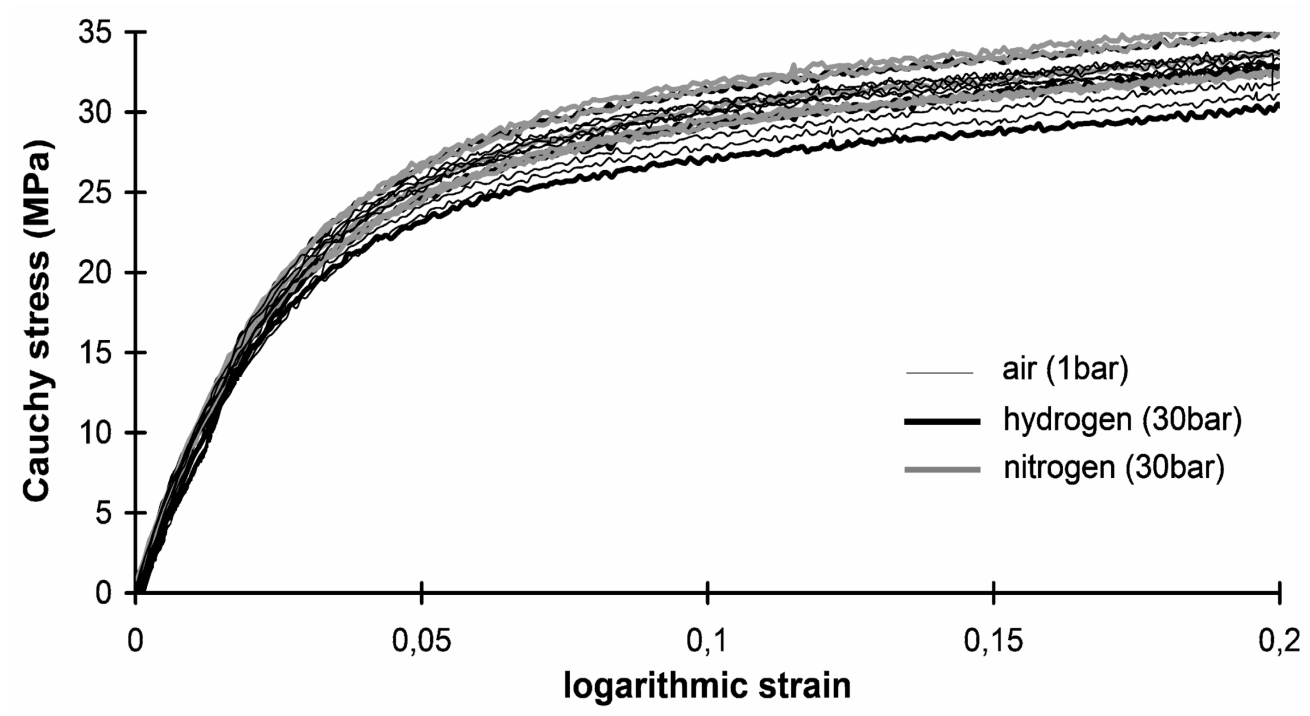

Fig. 1. Tensile behavior of as-received PE in air at atmospheric pressure, hydrogen at 30 bars and nitrogen at 30 bars.

By applying the time-temperature superposition principle, creep tests performed at 20, 40, 50, 60 and $80^{\circ} \mathrm{C}$ into atmorpheric air and into pressurized hydrogen could be used to build the two creep master-curves represented in Figure 2. Shift factors obtained from the master-curve building are plotted against $1 / \mathrm{T}$ in the insert. It is clear that the long term creep properties are not more influenced by hydrogen sorption than the tensile ones. Both shift factors series obey an Arrhenius activation law, as expected for this material in the $T \alpha_{c}$ temperature range. It suggests that hydrogen molecule is too small to affect molecular micro-mechanisms. 


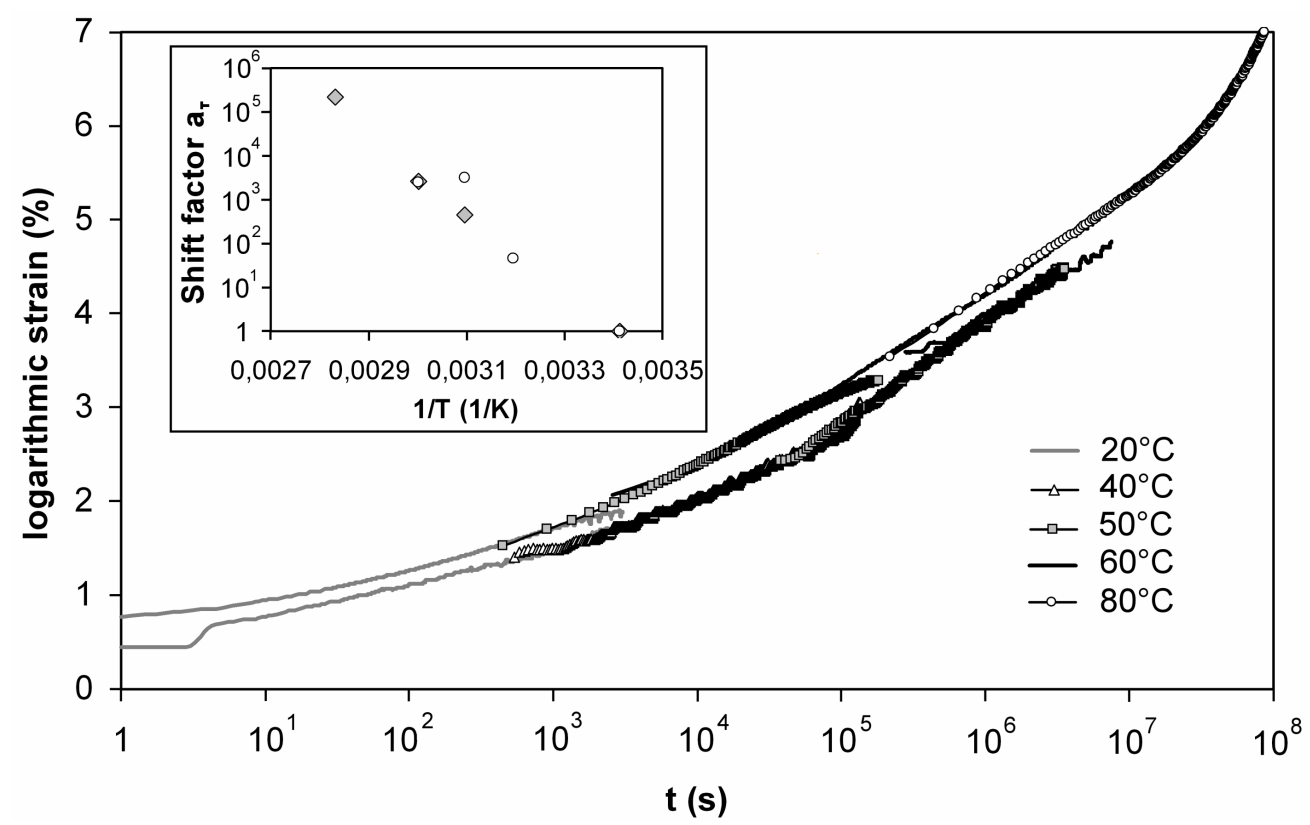

Fig. 2. Creep master curve at $20^{\circ} \mathrm{C}(5 \mathrm{MPa})$ in atmospheric air and into hydrogen at $30 \mathrm{bars}$, obtained by applying time temperature superposition principle to creep tests at $40,50,60$ and $80^{\circ} \mathrm{C}$. Insert: corresponding shift factors.

\section{Influence of long term aging into hydrogen atmosphere}

The goal was to evaluate the influence of aging parameters like temperature or hydrogen pressure upon the tensile properties, coupled or not to hydrogen diffusion. To this aim, series of samples aged in the conditions listed in Table 1 have been tested both in atmospheric air and pressurized hydrogen (30 bars). Materials issued from prior permeability tests and most specimens had undergone long aging durations.

Figure 3 plots the tensile stress-strain curves recorded for aged PE samples. Let us focus on the influence of hydrogen diffusion and mechanical behavior first, comparing tests performed in atmospheric air and 30 bars hydrogen for each aging history. After annealing above $\mathrm{T} \alpha_{\mathrm{c}}(\mathrm{V} 1$ and $\mathrm{V} 3$ ), ambient air curves tend to locate slightly above hydrogen curves, unlike after annealing below $\mathrm{T} \alpha_{c}$ (V2, V5 and V6). However, differences keep very slight, consistently with the discrepancy already depicted for as-received materials. Any effect of the aging pressure would arise from the comparison of V2 (20 bars) and V6 (5 bars) at $20^{\circ} \mathrm{C}$. The later curves appear slightly below the former ones but very close together however considering the tests reproducibility.

About the aging temperature influence, tests performed after aging at 5 bars show a rather clear higher stress level after aging at $80^{\circ} \mathrm{C}(\mathrm{V} 3)$ than after aging at $20^{\circ} \mathrm{C}(\mathrm{V} 6)$. However, this difference is no more observed after aging at 20 bars, since all curves overlap within a limited scatter range regardless the aging temperature of $20^{\circ} \mathrm{C}(\mathrm{V} 2), 50^{\circ} \mathrm{C}(\mathrm{V} 5)$ or $80^{\circ} \mathrm{C}(\mathrm{V} 1)$. 


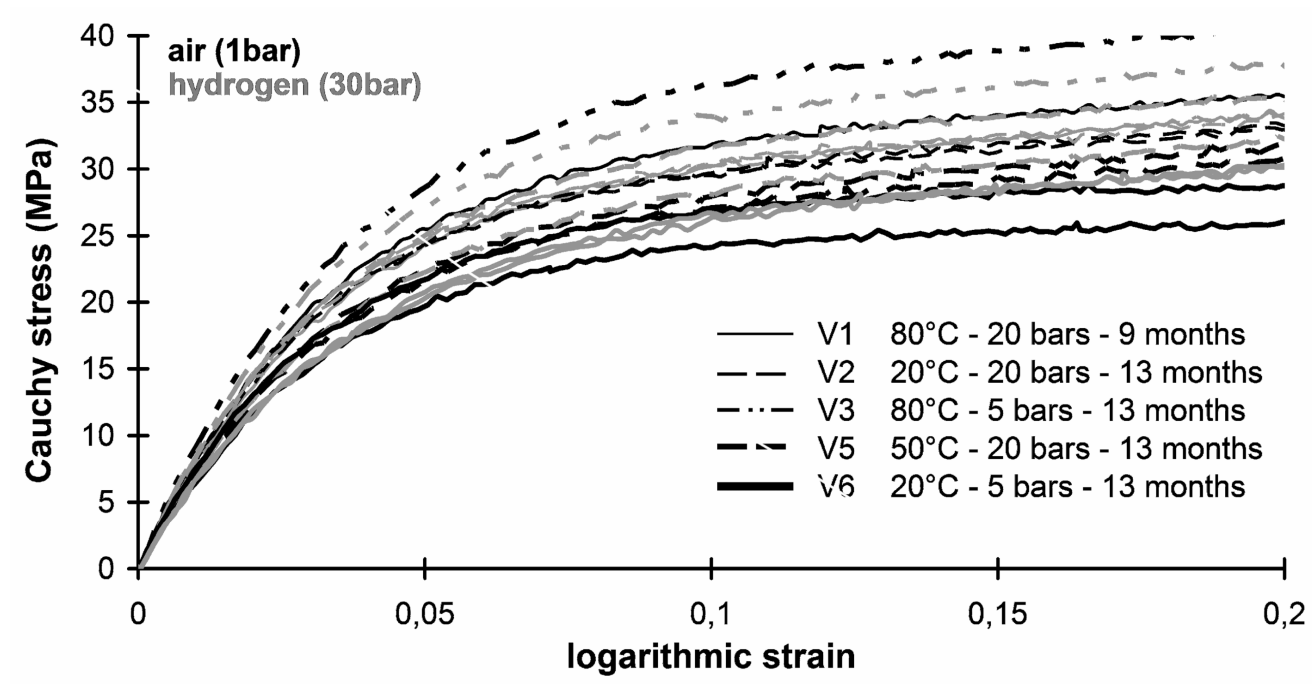

Fig. 3. Tensile curves obtained in $\mathrm{PE}$ in atmospheric air and 30 bars hydrogen, after various aging histories (see Table 1).

Despite slightly different strain-rates due to gauge length (i.e. $8.610^{-3} \mathrm{~s}^{-1}$ for as-received specimens against $1.610^{-2} \mathrm{~s}^{-1}$ for the aged ones), Figure 4 aims to highlight the influence of long term annealing by comparing the as-received data (curves from Figure 1) with the aged ones (curves from Figure 3). Considering that the increased experimental scatter observed in aged samples is highly linked to the very small size of samples, Figure 4 supports the weak influence of hydrogen diffusion coupling and aging processes.

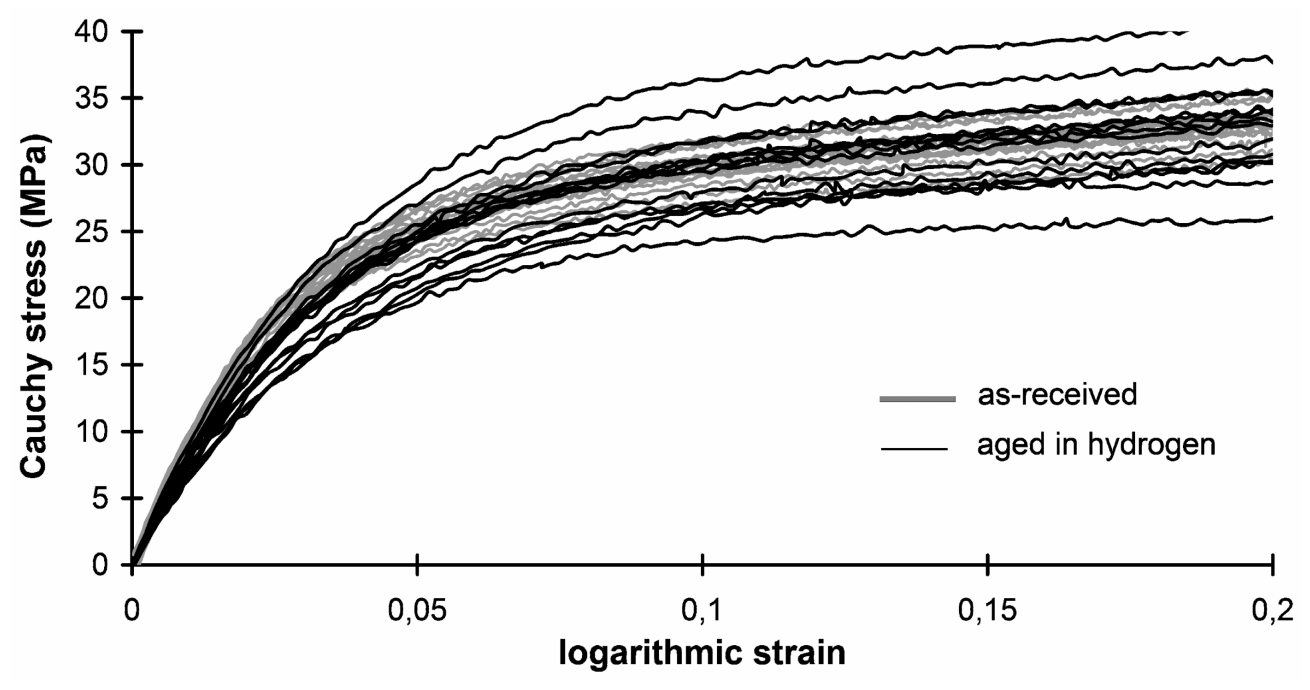

Fig. 4. Influence of aging history on the tensile behavior of PE: comparison between as-received samples from Figure 1 (thick grey line) and aged ones from Figure 3 (fine line). 


\section{CONCLUSION}

The mechanical behavior of polymers used in gaseous environment is bound to be affected by gas diffusion and mechanics coupling, and by aging processes due to long term gas exposure. Such issues have not been widely investigated, in particular for hydrogen atmosphere, mostly due to safety reasons associated with hydrogen manipulation. The purpose of this study was to provide new experimental data for the design of polymer parts undergoing hydrogen atmosphere, based on mechanical tensile experiments into pressurized hydrogen or nitrogen atmosphere. A polyethylene already used for natural gas network was investigated. The new experimental device developed here allows mechanical testing into pressurized hydrogen or nitrogen atmosphere, on small samples however. An unusual wide experimental scatter is enhanced by the associated small forces compared to the load cell capacity and by frictional forces due to the pressurization device. A more accurate measurement of the mechanical behavior description of a hydrogen pressure effect would need larger specimens, not easily compatible with the small volume of the chamber. Despite this experimental constraint and the subsequent wide scattering for tensile tests, several conclusions can be drawn.

Comparison between tests performed in atmospheric air and hydrogen at 30bars show that coupling between mechanics and hydrogen sorption is negligible. Both tensile properties (Young's modulus and yield stress) and long term creep behavior are unaffected by the gaseous atmosphere. After long-term aging up to more than one year in hydrogen atmosphere at various pressures $(5$ or 20 bars) and temperatures $\left(20^{\circ} \mathrm{C}, 50^{\circ} \mathrm{C}\right.$ and $\left.80^{\circ} \mathrm{C}\right)$, no deleterious effect was observed on the mechanical properties of polyethylene. Access to such data provides technological outcomes, e.g. guidelines for the design of structures dedicated to hydrogen atmosphere. For static properties at least, industrial applications involving these materials can be designed from the already known behavior, whatever for mono-layer or for multi-layered systems. Nevertheless, complementary studies must be carried out to investigate hydrogen effects on other phenomena like crack propagation for instance.

\section{ACKNOWLEDGEMENTS}

The experiments reported here were made with the support of the French National Agency for Research (ANR) (PolHYTube project; PAN-H program); authors wish to thank partners of the project: Arkema for providing the as-received materials, CEA-Grenoble for the aged samples, IMPINSA Lyon and IFP for permeability data.

\section{REFERENCES}

1. D.O. Stodilka, N.P. Kherani, W.T. Shmayda, S.J. Thorpe, Int J Hydrogen Energy 25, 1129 (2000)

2. S. Shishatskiy, C. Nistor, M. Popa, S.P. Nunes K.V. Peinemann, Adv Eng Mat 8, 390 (2006)

3. T.R. Cuthbert, N.J. Wagner, M.E. Paulaitis, G. Murgia, B. D'Aguanno, Macromol 32, 5017 (1999)

4. F. Piroux, E. Espuche, R. Mercier, J Membr Sci 22, 115 (2004)

5. V. Compan, M. Lopez-Lidon, A. Andrio, E. Riande, Macromol 31, 6984 (1998)

6. N.F.A. van der Vegt, Macromol 33, 3153 (2000)

7. L. Audouin, V. Langlois, J. Verdu, J.C.M. Debruijn, J Mat Sci 29, 569 (1994)

8. C.C. Hu, Y.J. Fu, S.W. Hsiao, K.R. Lee, J.Y. Lai, J Membr Sci 303, 29 (2007)

9. C.M. Laot, E. Marand, B. Schmittmann, R.K.P. Zia, Macromol 36, 8673 (2003)

10. C. Valançon, A. Roy, J.C. Grandidier, Oil \& Gas Sci and Technol - Rev IFP 61, 759 (2006)

11. J. Pocachard et al., Internal Report, ANR Program PAN'H-PolHYTube, 2009.

12. J. Crank. The mathematics of diffusion, Clarendon Press, Oxford, 1956.

13. M.H. Klopffer et al., Internal Report, ANR Program PAN'H-PolHYTube, 2009.

14. B. Flaconnèche, J. Martin, M.H. Klopffer, Oil \& Gas Sci and Technol - Rev. IFP 56, 261 (2001) 